\title{
XRCC3 T241M polymorphism and melanoma skin cancer risk: A meta-analysis
}

\author{
JINGHUA FAN $^{1}$, YUHUA FAN ${ }^{2}$, XIAOXIAO KANG $^{1}$ and LIMIN ZHAO ${ }^{1}$ \\ ${ }^{1}$ Department of Dermatology, Xi'an Central Hospital, Medical School of Xi'an Jiaotong University; \\ ${ }^{2}$ The First Department of Internal Medicine, The Fifth Hospital of Xi'an, Xi'an, Shaanxi 710003, P.R. China
}

Received June 7, 2014; Accepted February 25, 2015

DOI: 10.3892/ol.2015.3040

\begin{abstract}
Although the T241M polymorphism in the X-ray cross-complementing group 3 (XRCC3) gene has been implicated in the pathogenesis of melanoma, the results have been inconsistent. In this study, a meta-analysis was performed to assess the association of XRCC3 T241M polymorphism with melanoma. Published literature from PubMed and Embase databases was retrieved. The pooled odds ratio (OR) with 95\% confidence interval (CI) was calculated using fixed- or random-effects models. A total of six case-control studies containing 2,133 patients and 3,141 controls were enrolled into this meta-analysis. In a combined analysis, the results revealed no significant association between XRCC3 T241M polymorphism and melanoma risk in the overall population. In the subgroup analysis by ethnicity, no significant associations between the XRCC3 T241M polymorphism and melanoma risk were identified in Caucasians. However, when the analyses were restricted to three larger studies ( $\mathrm{n}>500$ cases), a significant association was noted with melanoma (TT vs. MT: $\mathrm{OR}=1.20,95 \% \mathrm{CI}=1.04-1.38$; dominant model: $\mathrm{OR}=0.86$, 95\% CI=0.75-0.98). In conclusion, the meta-analysis results suggest that the XRCC3 T241M polymorphism was associated with risk of melanoma. Further large and well-designed studies are needed to confirm this conclusion.
\end{abstract}

\section{Introduction}

Melanoma is a neoplastic lesion arising from epidermal melanocytes. It is estimated that 132,000 new cases of melanoma occur worldwide every year (1). According to the recent data from the International Agency for Research on Cancer (IARC), the highest reported national incidence rates for melanoma occurred in the populations of Australia (39 cases per 100,000

Correspondence to: Mr. Jinghua Fan, Department of Dermatology, Xi'an Central Hospital, Medical School of Xi'an Jiaotong University, 185 Houzaimen Street, Xi'an, Shaanxi 710003, P.R. China

E-mail: fanjinghua003@126.com

Key words: X-ray cross-complementing group 3, gene polymorphism, melanoma individuals per year) and New Zealand (34 cases per 100,000 individuals per year) (2). A previous study indicated that approximately $65-90 \%$ of all melanomas are attributable to ultraviolet radiation exposure (3). Ultraviolet radiation is subdivided into ultraviolet $\mathrm{A}$, ultraviolet $\mathrm{B}$ and ultraviolet $\mathrm{C}$. Of these, ultraviolet A wavelengths (320-400 nm) cause single-stranded breaks and DNA protein crosslinking, and generate free radicals that cause oxidative damage and DNA double-strand breaks (DSBs) (4). Ultraviolet B radiations (290-320 nm) induce damage in the form of cyclobutane pyrimidine dimers and pyrimidine photoproducts, which may lead to the formation of DSBs, chromosomal aberrations and recombination during the course of replication arrest (5).

To date, mammalian cells utilize four major DNA repair mechanisms to protect against genetic instability: base excision repair, mismatch repair, nucleotide excision repair and double-strand break repair (DSBR). DSBR is the most common form of radiation-induced DNA damage and includes both homologous recombination (HRR) and non-homologous end-joining (6). The X-ray repair cross-complementing group 3 (XRCC3) protein is involved in the HRR pathway to repair DNA damage and maintain genomic stability (7). The XRCC3 gene is localized on human chromosomes 14q32.3. The most frequent polymorphism in XRCC3 is a C/T transition resulting in an amino acid substitution from Thr to Met at codon 241 (T241M). In addition, variants of the T241M polymorphism may affect the function of the encoded protein and consequently alter the DNA repair capacity (8).

In recent years, several studies have been performed to evaluate the association between the T241M polymorphism and melanoma risk. However, the published results have been inconsistent. Meta-analysis is a useful tool in detecting an association that could otherwise remain masked in the sample size studies, particularly in those evaluating rare allele frequency polymorphisms (9). The aim of this meta-analysis was to investigate the association between $\mathrm{T} 241 \mathrm{M}$ polymorphism and susceptibility to melanoma using all eligible case-control studies published to date.

\section{Materials and methods}

Literature search. We searched for studies in the PubMed and Embase electronic databases using the terms 'melanoma', 'skin cancer', 'T241M', 'XRCC3', 'excision repair 
Table I. Characteristics of studies included in meta-analysis.

\begin{tabular}{|c|c|c|c|c|c|c|c|c|c|c|c|}
\hline \multirow[b]{2}{*}{ Study included (ref.) } & \multirow[b]{2}{*}{ Year } & \multirow[b]{2}{*}{ Area } & \multirow[b]{2}{*}{ Ethnicity } & \multirow[b]{2}{*}{ Cases/controls } & \multicolumn{3}{|c|}{$\begin{array}{l}\text { Genotypes of } \\
\text { cases }\end{array}$} & \multicolumn{3}{|c|}{$\begin{array}{l}\text { Genotypes of } \\
\text { controls }\end{array}$} & \multirow[b]{2}{*}{ HWE test } \\
\hline & & & & & TT & MT & MM & $\mathrm{TT}$ & MT & MM & \\
\hline Winsey et al (11) & 2000 & England & Caucasian & $125 / 211$ & 39 & 65 & 21 & 110 & 78 & 23 & 0.11 \\
\hline Duan et al (12) & 2002 & USA & Mixed & $305 / 319$ & 119 & 148 & 38 & 116 & 158 & 45 & 0.45 \\
\hline Figl et al (13) & 2010 & Germany & Caucasian & $1184 / 1274$ & 451 & 541 & 192 & 436 & 645 & 193 & 0.07 \\
\hline Gonçalves et al (14) & 2011 & Brazil & Mixed & $192 / 192$ & 78 & 89 & 25 & 95 & 79 & 18 & 0.79 \\
\hline Bertram et al (15) & 2004 & England & Caucasian & $140 / 335$ & 50 & 68 & 22 & 135 & 160 & 40 & 0.89 \\
\hline Han et al (16) & 2004 & USA & Mixed & $187 / 810$ & 75 & 84 & 28 & 300 & 396 & 114 & 0.36 \\
\hline
\end{tabular}

HWE, Hardy-Weinberg equilibrium.

cross-complementing group 3' and 'polymorphism'. The search was performed without any restrictions on language and was focused on studies conducted in humans. Further studies were identified by a hand search of references of original or review articles on this topic. If data or data subsets were published in more than one article, only the publication with the largest sample size was included.

Inclusion and exclusion criteria. Eligible studies included in the present analysis met the following criteria: i) studies that evaluated the association between the XRCC3 T241M polymorphism and melanoma, ii) a case-control study design, and iii) had detailed genotype frequency of cases and controls or could be calculated from the article text. The main exclusion criteria were: i) case reports, letters, reviews, meta-analyses and editorial articles, ii) non-case-control studies that evaluated the association between XRCC3 T241M polymorphism and melanoma risk, iii) studies in which the number of null and wild genotypes could not be ascertained, and iv) duplicate data were included in the studies.

Data extraction. Two investigators independently extracted data according to the inclusion criteria. Disagreement was resolved by discussion between them. If no consensus was reached, an expert was consulted to resolve the dispute and a final majority decision was made. For each study, the following data was collected: the first author's name, year of publication, country of origin, ethnicity, area, number of patients and controls, distributions of genotypes and alleles, and evidence of Hardy-Weinberg equilibrium (HWE). These are listed in Table I.

Data analysis. We tested whether genotype frequencies of controls were in HWE using the $\chi^{2}$ test. The odds ratio (OR) and corresponding $95 \%$ confidence interval (CI) were calculated to evaluate the association between the XRCC3 T241M polymorphism and melanoma risk under a homozygote comparison (TT vs. MM), a heterozygote comparison (TT vs. MT), a dominant model (MM+MT vs. TT) and a recessive mode (TT+MT vs. MM) between groups. Between-study heterogeneities were estimated using the $\mathrm{I}^{2}$ test. $\mathrm{I}^{2}$ values of 25 , 50 and $75 \%$ were defined as low, moderate and high estimates,

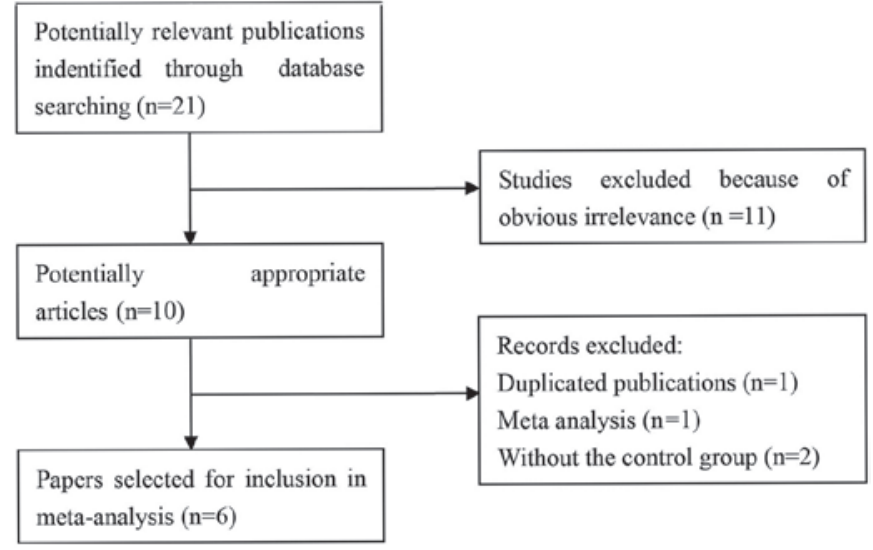

Figure 1. Flow chart of study selection based on inclusion and exclusion criteria.

respectively (10). If heterogeneity was observed among the studies, the pooled OR was estimated using the fixed-effects model $\left(\mathrm{P}>0.10\right.$ or $\left.\mathrm{I}^{2}<50 \%\right)$. Otherwise, the random-effects model was used to estimate the pooled OR. Subgroup analyses by ethnicity and sample size in cases were performed. Sensitivity analysis was performed by comparing the random-effect model values with the fixed-effect model values. Publication bias was investigated using funnel plot and Begg's funnel plot $(\mathrm{P}<0.05$ was considered to indicate a statistically significant difference). Analyses were performed with Stata software (version 12.0; Stata Corporation, College Station, TX, USA), using two-sided P-values.

\section{Results}

Identification of eligible studies. The search strategy retrieved 21 potentially relevant studies. Based on the inclusion criteria, six case-control studies with full text were included in this meta-analysis (11-16) and 15 studies were excluded. The flow chart for the study selection is summarized in Fig. 1. The six case-control studies selected included a total of 2,133 cases and 3,141 healthy controls. The HWE test was performed on genotype distribution of the controls; all of them were in HWE ( $>0.05)$. The studies had been carried out in England, 
Table II. Summary of odds ratios and 95\% confidence interval of T241M polymorphism with melanoma risk.

\begin{tabular}{|c|c|c|c|c|c|c|c|c|c|c|}
\hline \multirow[b]{2}{*}{ Subgroup } & \multirow[b]{2}{*}{ Genetic model } & \multicolumn{2}{|c|}{ Sample size } & \multirow[b]{2}{*}{ Type of model } & \multicolumn{2}{|c|}{$\begin{array}{c}\text { Test of } \\
\text { heterogeneity }\end{array}$} & \multicolumn{2}{|c|}{$\begin{array}{c}\text { Test of } \\
\text { association }\end{array}$} & \multicolumn{2}{|c|}{$\begin{array}{c}\text { Test of } \\
\text { publication bias }\end{array}$} \\
\hline & & Cases & Controls & & $\mathrm{I}^{2}$ & P-value & OR & $95 \% \mathrm{CI}$ & $\mathrm{Z}$ & P-value \\
\hline \multirow[t]{4}{*}{ Overall } & TT vs. MM & 2133 & 3141 & Random & $54.1 \%$ & 0.05 & 0.83 & $1.62-1.12$ & 0.24 & 0.81 \\
\hline & TT vs. MT & & & Random & $75.3 \%$ & 0.00 & 0.91 & $0.69-1.20$ & 0.24 & 0.81 \\
\hline & Dominant model & & & Random & $77.8 \%$ & 0.00 & 1.14 & $0.86-1.51$ & 0.24 & 0.81 \\
\hline & Recessive model & & & Fixed & $0.0 \%$ & 0.57 & 0.89 & $0.76-1.05$ & 0.24 & 0.81 \\
\hline \multirow[t]{4}{*}{ Caucasians } & TT vs. MM & 1449 & 1820 & Random & $74.6 \%$ & 0.02 & 0.70 & $0.39-1.24$ & 0.00 & 1.00 \\
\hline & TT vs. MT & & & Random & $88.2 \%$ & 0.00 & 0.80 & $0.44-1.46$ & 0.00 & 1.00 \\
\hline & Dominant model & & & Random & $89.1 \%$ & 0.00 & 1.31 & $0.72-2.38$ & 0.00 & 1.00 \\
\hline & Recessive model & & & Fixed & $0.0 \%$ & 0.39 & 0.86 & $0.71-1.05$ & 0.00 & 1.00 \\
\hline \multirow{4}{*}{$\begin{array}{l}\text { Sample size } \\
>500\end{array}$} & TT vs. MM & 1676 & 2403 & Fixed & $0.0 \%$ & 0.85 & 1.06 & $0.87-1.29$ & 1.04 & 0.30 \\
\hline & TT vs. MT & & & Fixed & $0.0 \%$ & 0.83 & 1.20 & $1.04-1.38$ & 1.04 & 0.30 \\
\hline & Dominant model & & & Fixed & $0.0 \%$ & 0.95 & 0.86 & $0.75-0.98$ & 1.04 & 0.30 \\
\hline & Recessive model & & & Fixed & $0.0 \%$ & 0.69 & 0.96 & $0.80-1.15$ & 1.04 & 0.30 \\
\hline
\end{tabular}

OR, odds ratio; CI, confidence interval.

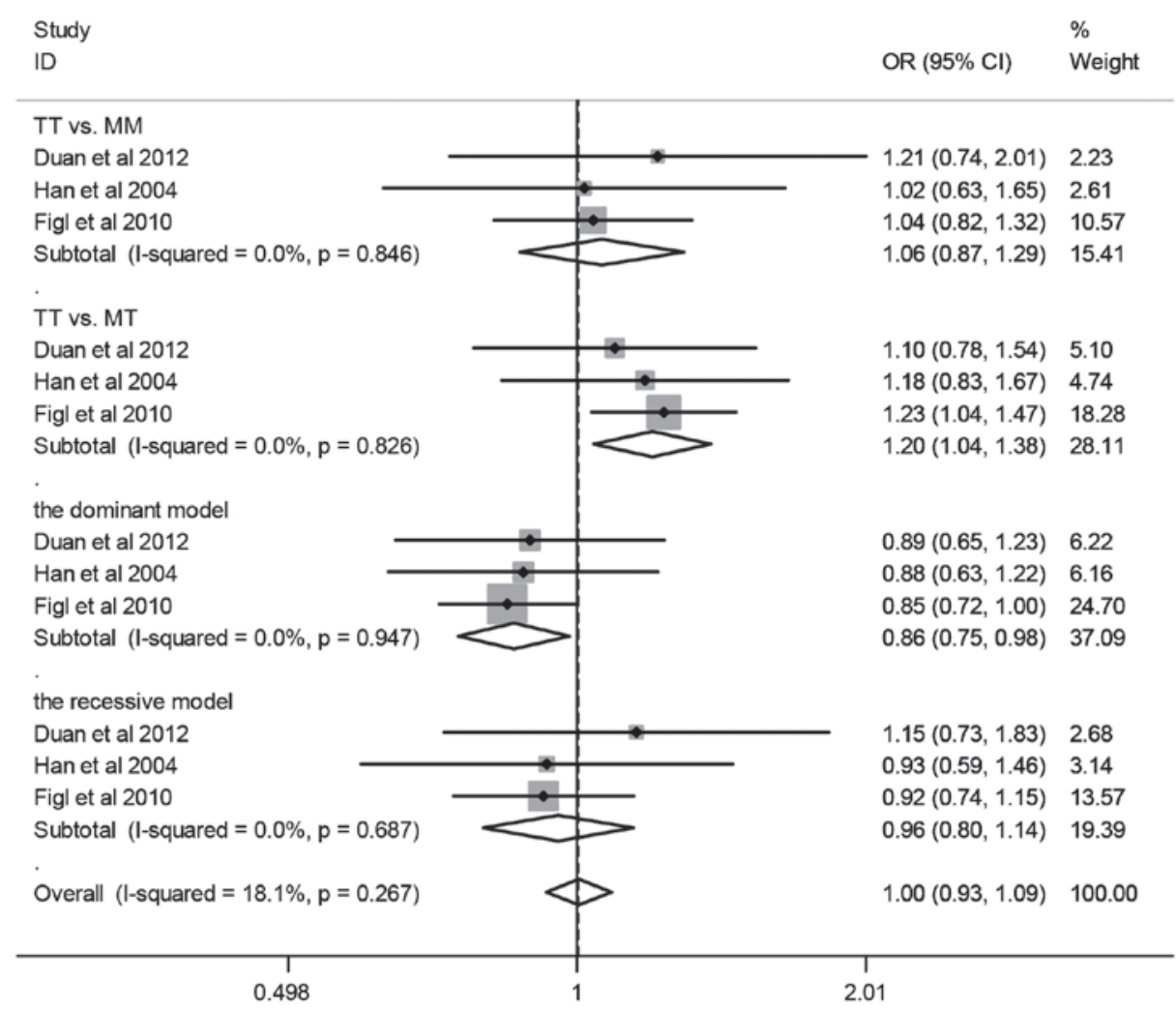

Figure 2. Meta-analysis of the association between X-ray cross-complementing group 3 gene T241M polymorphism and melanoma risk by sample size ( $>500)$.

USA, Germany and Brazil. Among them, there were three studies of Europeans $(11,13,15)$. The publishing year of the included studies ranged from 2000 to 2014. All of the articles were written in English. The source of controls was mainly based on healthy populations. Furthermore, the studies were conducted with $>500$ subjects included in the subgroup meta-analysis $(12,13,16)$. General characteristics and the allele and genotype distributions in the published articles included in this meta-analysis are shown in Table I.

Meta-analysis. A summary of the meta-analysis findings of the association between the XRCC3 T241M polymorphism and melanoma risk is shown in Fig. 2 and Table II. The combined results based on all studies revealed that variant genotypes 


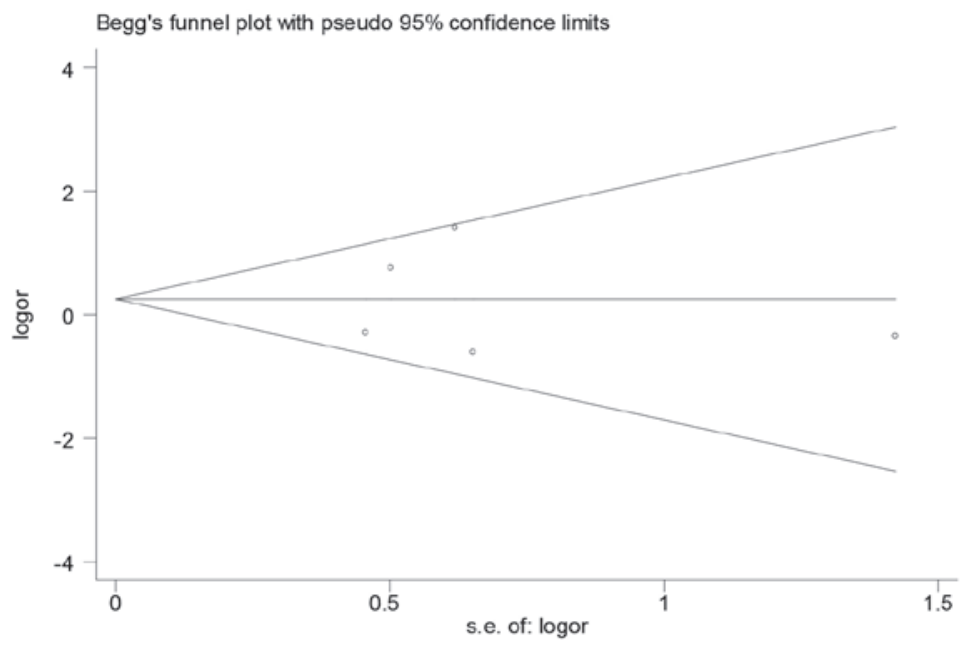

Figure 3. Begg's funnel plot test of publication bias for the association between X-ray cross-complementing group 3 gene T241M polymorphism and melanoma risk.

are not associated with increased melanoma risk in different genetic models (TT vs. MM: OR=0.92, 95\% $\mathrm{CI}=0.68-1.26$; TT vs. MT: $\mathrm{OR}=0.95,95 \% \mathrm{CI}=0.72-1.25$; dominant model: $\mathrm{OR}=1.07,95 \% \mathrm{CI}=0.81-1.41$; recessive model: $\mathrm{OR}=0.94$, $95 \% \mathrm{CI}=0.80-1.10)$. When stratified according to ethnicity, no significant association was detected in Caucasians (TT vs. MM: $\mathrm{OR}=0.70,95 \% \mathrm{CI}=0.39-1.24$; TT vs. $\mathrm{MT}: \mathrm{OR}=0.80,95 \%$ $\mathrm{CI}=0.44-1.46$; dominant model: $\mathrm{OR}=1.31,95 \% \mathrm{CI}=0.72-2.38$; recessive model: $\mathrm{OR}=0.86,95 \% \mathrm{CI}=0.71-1.05)$. In the stratified analysis by sample size (>500 subjects), we detected a significant association between T241M and melanoma (TT vs. MM: $\mathrm{OR}=1.06,95 \% \mathrm{CI}=0.87-1.29$; TT vs. $\mathrm{MT}$ : $\mathrm{OR}=1.20,95 \%$ $\mathrm{CI}=1.04-1.38$; dominant model: $\mathrm{OR}=0.86,95 \% \mathrm{CI}=0.75-0.98$; recessive model: $\mathrm{OR}=0.96,95 \% \mathrm{CI}=0.80-1.15)$. Sensitivity analyses were conducted by altering the statistic models. No material alteration was detected, indicating that our results were statistically robust.

Publication bias. The funnel plot and Begg's test were used to assess the publication bias of the literature. There was no evidence of publication bias in our study (Fig. 3). The results implied that the publication bias was low in the present meta-analysis (all $\mathrm{P}>0.05$ ). Information concerning the Begg's funnel plot is given in Table II.

\section{Discussion}

The incidence rate of cutaneous melanoma, which is etiologically linked to sun exposure, has rapidly grown over the years (17). Evidence suggests that cancer may be initiated by DNA damage, and that most DNA damage may be removed by DNA repair enzymes, including XRCC3. Numerous studies have evaluated the association of the XRCC3 T241M polymorphism with the risk of various cancer types, including colorectal, bladder, lung, breast and pancreatic cancer (18-20). A variety of studies have focused on the association between the XRCC3 T241M polymorphism and melanoma. However, the observed associations of these studies were inconclusive. The most likely reason for the inconsistencies among these studies is that they are single case-control studies with small sample sizes. To resolve these conflicting results, we conducted this meta-analysis to combine the same type of studies to increase the sample size and statistical power, and hence obtain a more reliable result.

The present meta-analysis, including 2,133 cases and 3,141 controls from six case-control studies, explored the association between the XRCC3 T241M polymorphism and melanoma risk. The results of the meta-analysis revealed that the $\mathrm{T} 241 \mathrm{M}$ polymorphism is not associated with increased or decreased risk of melanoma in the overall population. Considering that the result may be affected by ethnicity, an ethnicity-related subgroup analysis was performed, and no significant association was identified in Caucasians. Our meta-analysis involved several studies with small samples. There may be a selective bias for the correlation between the XRCC3 T241M polymorphism and melanoma development, so the association should be re-evaluated in studies with large sample sizes. When stratifying by sample size $(>500)$, this meta-analysis detected a significant association, suggesting the possibility of publication bias by smaller studies. Nevertheless, caution should be exercised when considering this conclusion. Sensitivity analysis was performed by comparing random-effect model values with fixed-effect values, and the results revealed that this meta-analysis was realistic and accurate. There was no evidence of publication bias in this meta-analysis (all $\mathrm{P}>0.05$ ).

The mechanism of how XRCC3 T241M polymorphism is associated with melanoma risk remains unclear. XRCC3 codes for a protein participating in HRR of DSB. It is a member of an emerging family of Rad-51-related proteins that may take part in homologous recombination to repair DSB and maintain chromosome stability (7), and the XRCC3 T241M polymorphism affects the DNA repair capacity of its encoded protein, and thus contributes to the development of melanoma (21). In addition, the potential influence of the XRCC3 T241M polymorphism may be affected by gene-gene and gene-environment interactions.

The present study has certain limitations. Firstly, there are only six studies included in our meta-analysis. More well-designed studies with large sample sizes are needed 
to further identify this association more comprehensively. Secondly, studies included in the present meta-analysis mainly provided data with regard to Caucasians, and other ethnicities including Asians and Africans should be investigated in future studies. Thirdly, subgroup analyses according to age, radiation exposure, histological types and other factors have not been performed due to insufficient relevant data available in the primary studies. Finally, only published English studies were included in this study, so publication and potential language biases may occur.

In conclusion, our meta-analysis indicates that XRCC3 $\mathrm{T} 241 \mathrm{M}$ polymorphism is associated with risk of melanoma. Large-scale case-control and population-based association studies are warranted to validate the risk identified in the current meta-analysis and investigate the potential gene-gene and gene-environment interactions on melanoma risk.

\section{References}

1. Foster PJ, Dunn EA, Karl KE, et al: Cellular magnetic resonance imaging: in vivo imaging of melanoma cells in lymph nodes of mice. Neoplasia 10: 207-216, 2008.

2. Matullo G, Palli D, Peluso M, et al: XRCC1, XRCC3, XPD gene polymorphisms, smoking and (32)P-DNA adducts in a sample of healthy subjects. Carcinogenesis 22: 1437-1445, 2001.

3. Glanz K, Buller DB and Saraiya M: Reducing ultraviolet radiation exposure among outdoor workers: state of the evidence and recommendations. Environ Health 6: 22, 2007.

4. Elmets $\mathrm{C}$ and Mukhtar H: Ultraviolet radiation and skin cancer: progress in pathophysiological mechanisms. Dermatology Foundation. Prog Dermatol 30: 1-16, 1996.

5. Lehmann A: Dual functions of DNA repair genes: molecular, cellular and clinical implications. Bioessays 20: 146-155, 1998

6. Altieri F, Grillo C, Maceroni M, et al: DNA damage and repair: from molecular mechanisms to health implications. Antioxid Redox Signal 10: 891-937, 2008.

7. Brenneman MA, Weiss AE, Nickoloff JA, et al: XRCC3 is required for efficient repair of chromosome breaks by homologous recombination. Mutat Res 459: 89-97, 2000.
8. Matullo G, Guarrera S and Carturan S: DNA repair gene polymorphisms, bulky DNA adducts in white blood cells and bladder cancer in a case-control study. Int J Cancer 92: 562-567, 2001.

9. Attia J, Thakkinstian A and D'Este C: Meta-analyses of molecular association studies: methodologic lessons for genetic epidemiology. J Clin Epidemiol 56: 297-303, 2003.

10. Higgins JP and Thompson SG: Quantifying heterogeneity in a meta-analysis. Stat Med 21: 1539-1558, 2002

11. Winsey SL, Haldar NA, Marsh HP, et al: A variant within the DNA repair gene XRCC3 is associated with the development of melanoma skin cancer. Cancer Res 60: 5612-5616, 2000.

12. Duan Z, Shen H, Lee JE, et al: DNA repair gene XRCC3 241 Met variant is not associated with risk of cutaneous malignant melanoma. Cancer Epidemiol Biomarkers Prev 11: 1142-1143, 2002.

13. Figl A, Scherer D, Nagore E, et al: Single-nucleotide polymorphisms in DNA-repair genes and cutaneous melanoma. Mutat Res 702: 8-16, 2010.

14. Gonçalves FT, Francisco G, de Souza SP, et al: European ancestry and polymorphisms in DNA repair genes modify the risk of melanoma: a case-control study in a high UV index region in Brazil. J Dermatol Sci 64: 59-66, 2011.

15. Bertram CG, Gaut RM, Barrett JH, et al: An assessment of a variant of the DNA repair gene XRCC3 as a possible nevus or melanoma susceptibility genotype. J Invest Dermatol 122: 429-432, 2004.

16. Han J, Colditz GA, Samson LD, et al: Polymorphisms in DNA double-strand break repair genes and skin cancer risk. Cancer Res 64: 3009-3013, 2004.

17. Leiter $U$ and Garbe C: Epidemiology of melanoma and nonmelanoma skin cancer-the role of sunlight. Adv Exp Med Biol 624: 89-103, 2008.

18. Jiang Z, Li C, Xu Y, et al: A meta-analysis on XRCC1 and XRCC3 polymorphisms and colorectal cancer risk. Int J Colorectal Dis 25: 169-180, 2010.

19. Jiao L, Hassan MM, Bondy ML, et al: XRCC2 and XRCC3 gene polymorphism and risk of pancreatic cancer. Am J Gastroenterol 103: 360-367, 2008.

20. Jin MJ, Chen K, Song L, et al: The association of the DNA repair gene XRCC3 Thr241Met polymorphism with susceptibility to colorectal cancer in a Chinese population. Cancer Genet Cytogenet 163: 38-43, 2005.

21. Araujo FD, Pierce AJ, Stark JM, et al: Variant XRCC3 implicated in cancer is functional in homology-directed repair of double-strand breaks. Oncogene 21: 4176-4180, 2002. 